\title{
Characteristics and Outcomes of Vertebrobasilar Artery Dissection with Accompanied Atherosclerosis
}

\author{
Chun Chien $^{a, b}$ Feng-Chi Chang ${ }^{b, c}$ Hui-Chi Huang ${ }^{a}$ Jui-Yao Tsai $^{a}$ \\ Chih-Ping Chung a, b \\ a Department of Neurology, Taipei Veterans General Hospital, Taipei, Taiwan; b National \\ Yang Ming University, Taipei, Taiwan; ' Department of Radiology, Taipei Veterans General \\ Hospital, Taipei, Taiwan
}

\author{
Keywords \\ Dissection · Vertebrobasilar disease $\cdot$ Posterior circulation stroke $\cdot$ Atherosclerosis
}

\begin{abstract}
Background: With the popularity of MRI use, vertebrobasilar artery dissection (VBD) has been found more frequently in patients with posterior circulation ischemic stroke (PCS). The relationship between VBD and atherosclerosis is unknown. The present study aimed to prove the hypothesis that PCS with pure VBD ( $p-V B D)$ and with VBD and accompanied cervical or cerebral artery atherosclerosis (a-VBD) have distinct manifestations. Methods: Patients with VBD-related PCS who were prospectively enrolled in the Taipei Veterans General Hospital Stroke Registry between January 1, 2010 and August 31, 2014 were recruited for the present study. Patients who had (1) atherosclerotic plaques with or without stenotic flow in cervical arteries on Duplex ultrasonography or (2) focal $>30 \%$ stenosis in cerebral arteries other than the dissecting region (usually in arterial bifurcations which are prone to atheroma formation) on brain MRA were defined as a-VBD. Results: There were 91 patients (67 [73.6\%] males, mean age 65.5 years $[S D=15.2$, range, 21-91]) with VBD-related $P C S$ recruited for the present study; 31 were $a-V B D$ and 60 were $p-V B D$. The results showed that there were significant differences in onset age, frequency of cigarette smoking, dissecting vascular involvement, and infarct locations between the 2 groups. In addition, compared with $p-V B D$, the a-VBD group had poorer functional recovery at 3 months and 1 year, respectively, which was independent of age, sex, vascular risk factors, stroke severity at admission, and treatment options. Conclusion: VBD-related PCS with and without accompanied atherosclerosis had different manifestations and should be regarded as distinct arterial diseases.




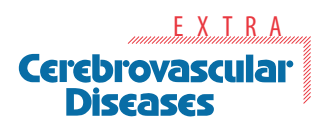

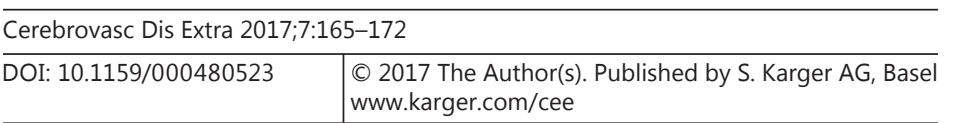

Chien et al.: Vertebrobasilar Artery Dissection and Atherosclerosis

\section{Introduction}

Vertebrobasilar artery dissection (VBD) is characterized by the intrusion of blood within the vascular layers (intramural hematoma) in the vertebral artery (VA) or/and basilar artery (BA) due to a tear in the intima of the vessel wall [1]. Magnetic resonance imaging (MRI) is proved to be a sensitive tool for the diagnosis of VBD due to its high-resolution imaging and direct visualization of intramural hematoma [2]. With the popularity of MRI use in acute ischemic stroke, VBD has been increasingly recognized as the etiology of posterior circulation ischemic stroke (PCS) [3-5]. Therefore, the characteristics and prognosis of patients with VBD-related PCS, whose numbers might have been underestimated in the past, need to be reassessed.

Atherosclerosis, a chronic inflammatory process, diffusely involves large- to mediumsized artery disease with atheromatous plaque formation in the arterial tunica intima and consequent artery lumen stenosis/occlusion [6]. The relationship between atherosclerosis and VBD has not yet been studied. The present study compared the clinical characteristics and prognosis of PCS induced by pure VBD ( $p$-VBD) and by VBD with accompanied cervical or cerebral artery atherosclerosis (a-VBD). We hypothesized that a-VBD might have distinct manifestations from $\mathrm{p}-\mathrm{VBD}$.

\section{Methods}

\section{Stroke Registry}

The Taipei Veterans General Hospital Stroke Registry has collected data for all patients with acute stroke admitted to the Emergency Room or Neurology Department wards since 2009. Standard investigations for acute ischemic stroke included high-resolution or standard brain MRI and MR arteriogram (MRA), Duplex ultrasound of neck arteries, color-coded transcranial Doppler, 24-h Holter monitor, and echocardiogram. Patient data were reviewed and a consensus concerning their stroke etiologies was reached by both neurologists and neuroradiologists. The following standardized criteria (shown by vascular studies) were used to classify the stroke etiology of VBD [5, 7-9]: (a) occlusion or stenosis of the VA or/and BA, (b) gradually tapered or/and long-segmental narrowing of the stenotic/occlusive vessel, and (c) intramural hematoma, intimal flap, or double lumen by high-resolution or standard MRI T1 sequence.

\section{Study Population}

The present study recruited patients with PCS induced by VBD who were registered in the Taipei Veterans General Hospital Stroke Registry between January 1, 2010 and August 31, 2014. We then classified these patients into 2 groups, a-VBD and p-VBD. Patients who had (1) atherosclerotic plaques with or without stenotic flow in cervical arteries (common and internal carotid arteries or proximal VA) on Duplex ultrasonography or (2) focal $>30 \%$ stenosis in cerebral arteries (internal carotid, middle, anterior, or posterior cerebral arteries, $\mathrm{VA}$ or $\mathrm{BA}$ ) other than the dissecting region (usually in arterial bifurcations which are prone to atheroma formation) on brain MRA were defined as a-VBD (Fig. 1) [10]. The other patients were classified as $\mathrm{p}$-VBD. Since previous studies have noticed a potential overestimation of the degree of stenosis by TOF MRA [11, 12], to avoid mistakenly classifying patients with the absence of atherosclerosis into the atherosclerosis group, we excluded patients with $0-29 \%$ stenosis shown on TOF MRA. Clinical characteristics including demographic data, vascular risk factor profile, symptoms/signs, stroke severity, involved vessel and brain regions, treatments during hospitalization, and functional outcomes at 3 months and 1 year, respectively, were compared between the 2 groups. Hypertension was defined as a self-report of a current antihypertensive medication prescription or as a measurement of systolic blood pressure $\geq 140 \mathrm{~mm} \mathrm{Hg}$ or diastolic blood pressure $\geq 90 \mathrm{~mm} \mathrm{Hg} \mathrm{[13].} \mathrm{DM} \mathrm{was} \mathrm{defined} \mathrm{as} \mathrm{a} \mathrm{self-report} \mathrm{of} \mathrm{current} \mathrm{DM} \mathrm{treatment} \mathrm{or} \mathrm{a}$ measurement of $\mathrm{HbA}_{1 \mathrm{c}} \geq 6.5 \%$ [14]. Hyperlipidemia was recorded if there was a self-report of use of statins or a blood level of total cholesterol $\geq 240 \mathrm{mg} / \mathrm{dL}$ [15]. Since the study population recruited VBD patients from the stroke registry, VBD without stroke was not included for the present study. The hospital's Institutional Review Board (IRB, Taipei Veterans General Hospital) approved the study procedures (IRB No. 2016-04$002 \mathrm{CC}$ ), and each participant provided informed consent. 


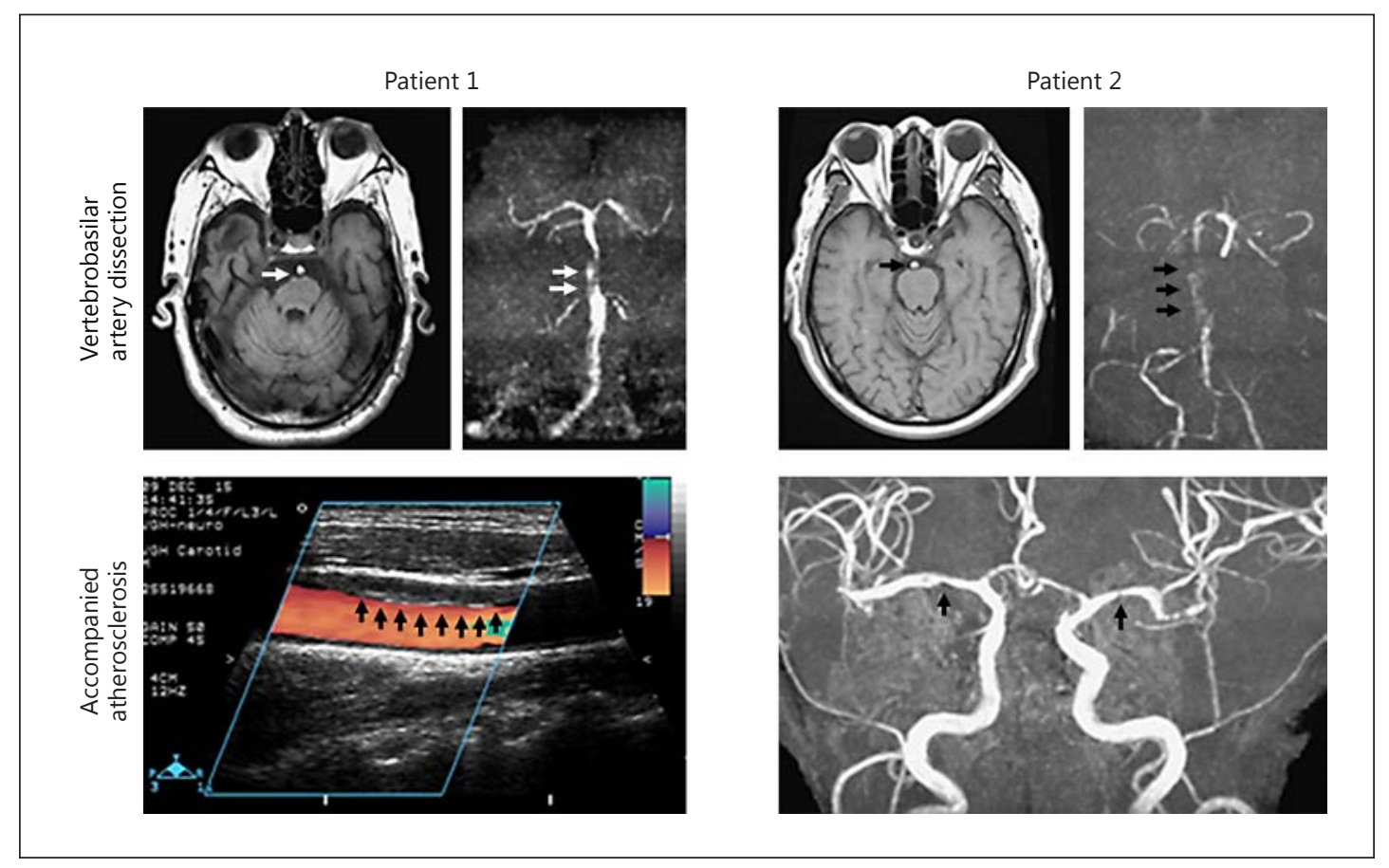

Fig. 1. Demonstration of vertebrobasilar artery dissection with accompanied atherosclerosis. Two patients with basilar artery dissection (upper arrows) and accompanied atherosclerosis evident on carotid artery ultrasonography (atherosclerosis plaque, left lower arrows) and on magnetic resonance angiography (atherosclerotic stenosis, right lower arrows), respectively.

\section{Brain MRI Acquisition}

All patients were imaged with a 1.5-T MRI scanner (Excite II; GE Medical Systems, Milwaukee, WI, USA) during hospitalization. Cranial 3D TOF MRA without magnetization transfer contrast was performed to detect the presence of focal narrowing in cerebral arteries. The severity of stenosis was determined with the ratio of arterial lumen at focal narrowing and arterial lumen at adjacent proximal part (lumen focal narrowing/ lumen proximal part $\times 100 \%$.

\section{Duplex Ultrasonography of Neck Arteries}

All patients underwent ultrasound imaging of the bilateral carotid and vertebral arteries (V0-V2) during hospitalization (Sonos 5500; Hewlett Packard, Andover, MA, USA). Assessment of plaque was undertaken using a high-resolution, linear array 2D transducer and scanning the artery in cross-section (carotid arteries) and longitudinal view (proximal vertebral arteries). Plaque was defined as local thickening of the intima-media thickness $>50 \%$ compared with the surrounding vessel wall, an intima-media thickness $>1.5$ $\mathrm{mm}$, or local thickening $>0.5 \mathrm{~mm}[16]$.

\section{Statistical Analyses}

Analyses were performed with SAS software, version 9.1 (SAS Institute, Cary, NC, USA). All values were expressed as mean (SD) for continuous variables and as number (percentage) for discrete variables. The nonparametric Wilcoxon rank-sum test was used for group comparisons. The $\chi^{2}$ test or the Fisher exact test was performed for categorical variables as appropriate. To test whether the presence of atherosclerosis was associated with poor functional outcomes (modified Rankin Scale $\geq 3$ ) at 3 months and 1 year, we used multivariate regression analyses, and odds ratios with 95\% confidence interval were calculated. Variables such as age, sex, vascular risk factors (hypertension, diabetes mellitus, hyperlipidemia, and cigarette smoking), stroke severity at admission (NIH Stroke Scale), and treatment option were adjusted in multivariate analyses. In our previous VBD study, the median modified Rankin Scale score at 3 months was 3 [17]; therefore, we used it as the cutoff point. 
Table 1. Demographic characteristics and presentation at admission in vertebrobasilar artery dissection with and without accompanied atherosclerosis

\begin{tabular}{lccr}
\hline & \multicolumn{2}{l}{ Atherosclerosis } & $p$ \\
\cline { 2 - 3 } & presence $(n=31)$ & absence $(n=60)$ & \\
\hline Age, years & $75.8(8.6)$ & $60.2(15.1)$ & $<0.001$ \\
Male & $20(30.9 \%)$ & $47(69.1 \%)$ & 0.313 \\
Vascular risk factors & & & 0.609 \\
$\quad$ Hypertension & $25(80.6 \%)$ & $45(75.0 \%)$ & 0.125 \\
Diabetes mellitus & $19(61.3 \%)$ & $26(43.3 \%)$ & 0.179 \\
Hyperlipidemia & $15(48.4 \%)$ & $20(33.3 \%)$ & 0.033 \\
Cigarette smoking & $5(16.1 \%)$ & $23(38.3 \%)$ & 0.184 \\
$\quad$ Body weight mass & $24.5(3.1)$ & $25.4(3.4)$ & 0.548 \\
Presentation at admission & 0 & $3(5.0 \%)$ & 0.971 \\
Associated trauma history & $7.6(6.9)$ & $7.6(9.2)$ & 0.279 \\
$\quad$ NIH Stroke Scale & $4(12.9 \%)$ & $14(23.3 \%)$ & 0.658 \\
Headache & $1(3.2 \%)$ & $4(6.7 \%)$ & \\
Neck pain & & & \\
\hline
\end{tabular}

Data are presented as mean (SD) or number of patients (percentage).

\section{Results}

There were 91 patients (67 [73.6\%] males, mean age 65.5 years [SD $=15.2$, range, 21-91]) with VBD-related PCS recruited for the present study; 31 were a-VBD and 60 were p-VBD. Comparisons of demographic characteristics and presentation at admission between a-VBD and p-VBD are presented in Table 1. Patients in the a-VBD group were older than those in the $\mathrm{p}$-VBD group. Both groups had a similar frequency of vascular risk factors except cigarette smoking, which was more frequent in the p-VBD group. Stroke severity (NIH Stroke Scale) was similar between the groups. There was a trend that patients in the $\mathrm{p}$-VBD group had more frequent headache and neck pain at presentation than those in the a-VBD group.

Table 2 demonstrates the vascular involvement, infarct regions, management strategy, and outcomes during hospitalization in patients with VBD-related PCS. Both the a-VBD and p-VBD groups had more frequent intracranial than extracranial artery involvement. However, there was more frequent bilateral intracranial VA and BA involvement in the a-VBD group than in the p-VBD group. Extracranial VA involvement in the p-VBD group was twice as frequent as that in the a-VBD group, though this was statistically nonsignificant. Correspondingly, the infarct regions were more frequent over the middle posterior circulation region (pons or/and anterior inferior cerebellar artery-supplied territory) in the a-VBD group than in the p-VBD group, and over the proximal posterior circulation region (medulla or/and posterior inferior cerebellar artery-supplied territory) in the p-VBD group than in the a-VBD group. The results also showed that though clinical worsening occurred more frequently in the a-VBD group, mortality in the p-VBD group was twice as frequent as that in the a-VBD group; both differences were statistically nonsignificant .

Table 2 also shows that functional outcomes were significantly different between the 2 groups; the modified Rankin Scale score at 3 months and 1 year, respectively, was lower in the p-VBD group compared with the a-VBD group. To adjust for confounding factors, multivariate analyses were performed (Table 3 ). The results showed that the presence of accom- 
Table 2. Clinical manifestations in vertebrobasilar artery dissection with and without accompanied atherosclerosis

\begin{tabular}{|c|c|c|c|}
\hline & \multicolumn{2}{|l|}{ Atherosclerosis } & \multirow[t]{2}{*}{$p$} \\
\hline & presence $(n=31)$ & absence $(n=60)$ & \\
\hline \multicolumn{4}{|l|}{ Dissection involvement } \\
\hline Extracranial vertebral artery & $4(12.9 \%)$ & $14(23.3 \%)$ & 0.279 \\
\hline \multicolumn{4}{|l|}{ Intracranial vertebral artery } \\
\hline Unilateral & $17(54.8 \%)$ & $31(51.7 \%)$ & 0.827 \\
\hline Bilateral & $7(22.6 \%)$ & $6(10.0 \%)$ & 0.067 \\
\hline Basilar artery & $30(96.8 \%)$ & $45(75.0 \%)$ & 0.007 \\
\hline \multicolumn{4}{|l|}{ Infarct region $^{\mathrm{a}}$} \\
\hline Distal posterior circulation & $7(22.6 \%)$ & $16(26.7 \%)$ & 0.801 \\
\hline Middle posterior circulation & $26(83.9 \%)$ & $32(53.3 \%)$ & 0.005 \\
\hline Proximal posterior circulation & $8(25.8 \%)$ & $28(46.7 \%)$ & 0.071 \\
\hline \multicolumn{4}{|l|}{ Management } \\
\hline Antiplatelets & 27 (87.1\%) & $39(65.0 \%)$ & 0.150 \\
\hline Anticoagulants & $4(12.9 \%)$ & $21(35.0 \%)$ & 0.150 \\
\hline Stenting & $1(3.2 \%)$ & $3(5.0 \%)$ & 1.000 \\
\hline \multicolumn{4}{|l|}{ During hospitalization } \\
\hline Clinical worsening & $7(22.6 \%)$ & $8(13.3 \%)$ & 0.371 \\
\hline Mortality & $1(3.2 \%)$ & $4(6.7 \%)$ & 0.658 \\
\hline \multicolumn{4}{|l|}{ Modified Rankin Scale } \\
\hline At 3 months & $3.2(1.7)$ & $2.2(2.0)$ & 0.020 \\
\hline At 1 year & $2.8(2.0)$ & $1.6(2.2)$ & 0.008 \\
\hline \multicolumn{4}{|c|}{$\begin{array}{l}\text { Data are presented as mean (SD) or number of patients (percentage). }{ }^{a} \text { Infarct locations were categorized } \\
\text { as involving proximal (medulla or/and posterior inferior cerebellar artery-supplied territory), middle (pons } \\
\text { or/and anterior inferior cerebellar artery-supplied territory), or/and distal (midbrain, posterior cerebral } \\
\text { artery, or/and superior cerebellar artery-supplied territory) posterior circulation regions. }\end{array}$} \\
\hline
\end{tabular}

Table 3. Short- and long-term functional outcomes in vertebrobasilar artery dissection with accompanied atherosclerosis

\begin{tabular}{lrrr}
\hline The presence of accompanied atherosclerosis & OR & $95 \%$ CI & $p^{*}$ \\
\hline Modified Rankin Scale $\geq 3$ at 3 months & 6.4 & $1.2-35.4$ & 0.033 \\
Modified Rankin Scale $\geq 3$ at 1 year & 10.6 & $1.5-76.5$ & 0.019 \\
\hline
\end{tabular}

* Adjusted for age, sex, vascular risk factors (hypertension, diabetes mellitus, hyperlipidemia and cigarette smoking), stroke severity at admission (NIH Stroke Scale), and treatment options.

panied atherosclerosis in VBD-related PCS was significantly associated with a poorer functional outcome at 3 months and 1 year, respectively, which was independent of age, sex, vascular risk factors, stroke severity at admission, and treatment options.

\section{Discussion}

The main finding of our study was that PCS induced by VBD with and without accompanied cervical or/and cerebral artery atherosclerosis had different manifestations. Significant differences included onset age, frequency of cigarette smoking, dissecting vascular 
involvement, infarct locations, and functional outcomes at 3 months and 1 year. The results suggest that VBD with and without accompanied atherosclerosis might have distinct pathophysiologies.

Several clinical manifestations of a-VBD shown in the present study were compatible with systemic atherosclerosis; there were more elderly people, more diffusely vascular involvement (bilateral VA and BA involvement), more frequent clinical worsening at the acute stage, and poorer functional recovery at 3 months and 1 year, respectively, in a-VBD [5, $10,18]$. Dissection has been described in aorta atherosclerotic atheroma by pathological studies [19]. Ulceration of an atheromatous plaque in the aorta may disrupt the internal elastic lamina, extend into the media, and result in intramural hematoma formation. However, the relationship between VBD and atherosclerosis has been less studied, both clinically and pathologically. The classification method used in the present study would need future studies such as molecular imaging or pathological assessment to validate a possible underlying atherosclerosis pathology in the vessel walls of VBD in a-VBD patients [10].

Patients with PCS induced by VBD without accompanied cervical or cerebral artery atherosclerosis in the present study were younger, had more frequent cigarette smoking habits (besides several other vascular risk factors), headache and neck pain at presentation, and mortality at an early stage, and better functional recovery at 3 months and 1 year. These features were consistent with previous acknowledgments of ischemic stroke induced by VBD, affecting younger people, usually with 1 fetal event, but with better functional recovery in survivors $[1,20-23]$.

Consistent with our result, a Finnish study also found a higher frequency of cigarette smoking in patients with cervicocerebral artery dissection than in the general population [24]. In addition, our previous study showed that cigarette smoking is associated with poorer 1-year functional outcome in intracranial VBD-related PCS [17]. These results suggest a role of cigarette smoking in the pathophysiology of VBD. Chronic exposure to cigarette smoking would cause vasoconstriction via activation of the sympathetic system, as well as endothelial dysfunction via oxidative stress and inflammatory mechanisms [25-27]. Moreover, cigarette smoking elicits a marked activation of leukocytes and platelets which could also lead to vascular damages $[26,27]$. This smoking-related vascular impairment might make individuals more susceptible to artery dissection. Meanwhile, a recent study has shown that the functions of endothelial progenitor cells, which are required for vascular repair after endothelial injury, are impaired in cigarette smokers [28]. Dysfunctional endothelial progenitor cells might be unable to heal injured vessel structures after minor trauma and lead to artery dissection. We will need further studies to validate these postulations.

There were limitations in the present study. The results might only be applied to Asian patients, in whom (compared with Western patients) intracranial VBD was more frequently reported than extracranial VA dissection [20, 29-31]. Besides, our classification methods using the presence or absence of atherosclerosis plaque or stenosis on imaging might not be able to rule out a possible subclinical atherosclerosis pathophysiology in patients with p-VBD. Our results might only find a relationship between evident atherosclerosis (instead of the presence of an underlying atherosclerosis pathology) and VBD-related PCS. Lastly, the present observational study did not provide clues to management strategy regarding the presence of atherosclerosis in VBD-related PCS. However, our results suggest that a future trial for the appropriate management of VBD should consider whether there is a presence of accompanied atherosclerosis in patients with VBD.

This is the first report discussing the relationship between VBD and atherosclerosis. VBD-related PCS with and without accompanied atherosclerosis had different manifestations and should be regarded as distinct arterial diseases. Their functional outcomes and management strategy should be considered separately. 


\section{Cerebrovascular Diseases}

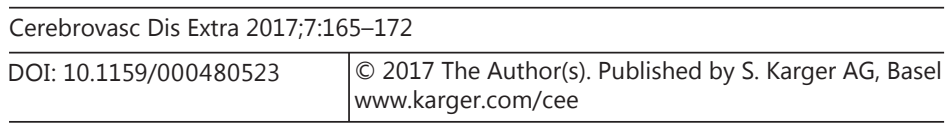

Chien et al.: Vertebrobasilar Artery Dissection and Atherosclerosis

\section{Disclosure Statement}

All authors report no conflicts of interest.

\section{Funding Sources}

Dr. Chung was supported by the Ministry of Science and Technology and Taipei Veterans General Hospital, Taiwan (VGH V105C-055; MOST 104-2314-B-075-MY3).

\section{References}

1 Caplan LR, Baquis GD, Pessin MS, D’Alton J, Adelman LS, DeWitt LD, Ho K, Izukawa D, Kwan ES: Dissection of the intracranial vertebral artery. Neurology 1988;38:868-877.

-2 Kitanaka C, Tanaka J, Kuwahara M, Teraoka A: Magnetic resonance imaging study of intracranial vertebrobasilar artery dissections. Stroke 1994;25:571-575.

3 Chung CP: Types of stroke and their differential diagnosis; in Caplan LR, Biller J, Leary MC, Lo EH, Thomas AJ, Yenari M, Zhang JH (eds): Primer on Cerebrovascular Diseases, ed 2. San Diego, Academic Press, 2017, pp 372-376.

4 von Sarnowski B, Schminke U, Grittner U, Tanislav C, Böttcher T, Hennerici MG, Tatlisumak T, Putaala J, Kaps M, Fazekas F, Enzinger C, Rolfs A, Kessler C; sifap1 Investigators: Posterior versus anterior circulation stroke in young adults: a comparative study of stroke aetiologies and risk factors in stroke among young Fabry patients (sifap1). Cerebrovasc Dis 2017;43:152-160.

5 Chung CP, Yong CS, Chang FC, Sheng WY, Huang HC, Tsai JY, Hsu HY, Hu HH: Stroke etiology is associated with outcome in posterior circulation stroke. Ann Clin Transl Neurol 2015;2:510-517.

-6 Tabas I, García-Cardeña G, Owens GK: Recent insights into the cellular biology of atherosclerosis. J Cell Biol 2015;209:13-22.

7 Caplan LR, Wityk RJ, Glass TA, Tapia J, Pazdera L, Chang HM, Teal P, Dashe JF, Chaves CJ, Breen JC, Vemmos K, Amarenco P, Tettenborn B, Leary M, Estol C, Dewitt LD, Pessin MS: New England Medical Center Posterior Circulation registry. Ann Neurol 2004;56:389-398.

-8 Caplan LR, Chung CS, Wityk R, Glass T, Tapia J, Pazdera L, Chang HM, Dashe J, Chaves C, Vemmos K, Leary M, Dewitt L, Pessin M: New England medical center posterior circulation stroke registry. I. Methods, data base, distribution of brain lesions, stroke mechanisms, and outcomes. J Clin Neurol 2005;1:14-30.

-9 Park KJ, Jung SC, Kim HS, Choi CG, Kim SJ, Lee DH, Suh DC, Kwon SU, Kang DW, Kim JS: Multi-contrast highresolution magnetic resonance findings of spontaneous and unruptured intracranial vertebral artery dissection: qualitative and quantitative analysis according to stages. Cerebrovasc Dis 2016;42:23-31.

10 Owen DR, Lindsay AC, Choudhury RP, Fayad ZA: Imaging of atherosclerosis. Annu Rev Med 2011;62:25-40.

-11 Pan XM, Saloner D, Reilly LM, Bowersox JC, Murray SP, Anderson CM, Gooding GA, Rapp JH: Assessment of carotid artery stenosis by ultrasonography, conventional angiography, and magnetic resonance angiography: correlation with ex vivo measurement of plaque stenosis. J Vasc Surg 1995;21:82-88.

12 Nederkoorn PJ, Elgersma OE, Mali WP, Eikelboom BC, Kappelle LJ, van der Graaf Y: Overestimation of carotid artery stenosis with magnetic resonance angiography compared with digital subtraction angiography. J Vasc Surg 2002;36:806-813.

13 Jones DW, Hall JE: Seventh report of the Joint National Committee on Prevention, Detection, Evaluation, and Treatment of High Blood Pressure and evidence from new hypertension trials. Hypertension 2004;43:1-3.

14 American Diabetes Association: Diagnosis and classification of diabetes mellitus. Diabetes Care 2010;33(suppl 1):S62-S69.

15 National Cholesterol Education Program (NCEP) Expert Panel on Detection, Evaluation, and Treatment of High Blood Cholesterol in Adults (Adult Treatment Panel III): Third Report of the National Cholesterol Education Program (NCEP) Expert Panel on Detection, Evaluation, and Treatment of High Blood Cholesterol in Adults (Adult Treatment Panel III) final report. Circulation 2002;106:3143-3421.

-16 Touboul PJ, Hennerici MG, Meairs S, Adams H, Amarenco P, Bornstein N, Csiba L, Desvarieux M, Ebrahim S, Hernandez Hernandez R, Jaff M, Kownator S, Naqvi T, Prati P, Rundek T, Sitzer M, Schminke U, Tardif JC, Taylor A, Vicaut E, Woo KS: Mannheim carotid intima-media thickness and plaque consensus (2004-2006-2011). An update on behalf of the advisory board of the 3rd, 4th and 5th watching the risk symposia, at the 13th, 15th and 20th European Stroke Conferences, Mannheim, Germany, 2004, Brussels, Belgium, 2006, and Hamburg, Germany, 2011. Cerebrovasc Dis 2012;34:290-296.

17 Chung CP, Chang FC, Huang HC, Tsai JY, Lin YY: Impacts of cigarette smoking and basilar artery flow on 1-year recovery in 3-month survivors of intracranial vertebrobasilar artery dissection-related ischemic stroke. Acta Neurol Taiwan 2016;25:75-82. 
18 Yamamoto H, Bogousslavsky J, van Melle G: Different predictors of neurological worsening in different causes of stroke. Arch Neurol 1998;55:481-486.

19 Movsowitz HD, Lampert C, Jacobs LE, Kotler MN: Penetrating atherosclerotic aortic ulcers. Am Heart J 1994; 128:1210-1217.

20 Kim BM, Kim SH, Kim DI, Shin YS, Suh SH, Kim DJ, Park SI, Park KY, Ahn SS: Outcomes and prognostic factors of intracranial unruptured vertebrobasilar artery dissection. Neurology 2011;76:1735-1741.

-21 Arnold M, Bousser MG, Fahrni G, Fischer U, Georgiadis D, Gandjour J, Benninger D, Sturzenegger M, Mattle HP, Baumgartner RW: Vertebral artery dissection: presenting findings and predictors of outcome. Stroke 2006; 37:2499-2503.

22 Ali MS, Amenta PS, Starke RM, Jabbour PM, Gonzalez LF, Tjoumakaris SI, Flanders AE, Rosenwasser RH, Dumont AS: Intracranial vertebral artery dissections: evolving perspectives. Interv Neuroradiol 2012;18: 469-483.

23 de Bray JM, Penisson-Besnier I, Dubas F, Emile J: Extracranial and intracranial vertebrobasilar dissections: diagnosis and prognosis. J Neurol Neurosurg Psychiatry 1997;63:46-51.

24 Metso TM, Metso AJ, Salonen 0, Haapaniemi E, Putaala J, Artto V, Helenius J, Kaste M, Tatlisumak T: Adult cervicocerebral artery dissection: a single-center study of 301 Finnish patients. Eur J Neurol 2009;16:656-661.

-25 Csiszar A, Podlutsky A, Wolin MS, Losonczy G, Pacher P, Ungvari Z: Oxidative stress and accelerated vascular aging: implications for cigarette smoking. Front Biosci (Landmark Ed) 2009;14:3128-3144.

26 Powell JT: Vascular damage from smoking: disease mechanisms at the arterial wall. Vasc Med 1998;3:21-28.

27 Leone A, Landini L: Vascular pathology from smoking: look at the microcirculation! Curr Vasc Pharmacol 2013;11:524-530.

28 Paschalaki KE, Starke RD, Hu Y, Mercado N, Margariti A, Gorgoulis VG, Randi AM, Barnes PJ: Dysfunction of endothelial progenitor cells from smokers and chronic obstructive pulmonary disease patients due to increased DNA damage and senescence. Stem Cells 2013;31:2813-2826.

29 Huang YC, Chen YF, Wang YH, Tu YK, Jeng JS, Liu HM: Cervicocranial arterial dissection: experience of 73 patients in a single center. Surg Neurol 2009;72(suppl 2):S20-S27.

-30 Tsukahara T, Minematsu K: Overview of spontaneous cervicocephalic arterial dissection in Japan. Acta Neurochir Suppl 2010;107:35-40.

-31 Chang FC, Yong CS, Huang HC, Tsai JY, Sheng WY, Hu HH: Posterior circulation ischemic stroke caused by arterial dissection: characteristics and predictors of poor outcomes. Cerebrovasc Dis 2015;40:144-150. 